\title{
Un antropólogo en el museo: reflexiones a partir de la experiencia de la profesionalización de la antropología en los museos colombianos
}

\author{
HECTOR GARCÍA BOTERO \\ Museo del Oro, Banco de la República | Bogotá, Colombia \\ hgarcibo@banrep.gov.co
}

DOI 10.11606/issn.2316-9133.v30i1pe187609

resumen La presencia de la antropología en los museos tiene una larga tradición, tanto en las tradiciones hegemónicas como subalternas de la disciplina. Desde finales del siglo XX, el tratamiento de las colecciones de "los otros" de la modernidad, históricamente ligado a la experticia de los antropólogos, ha sido objeto de cuestionamiento crítico desde distintos lugares, tanto académicos como no-académicos. Este texto presenta una mirada personal sobre esos asuntos para proponer una forma específica de hacer y pensar la antropología en los museos.

palabras clave Museos. Profesionalización de la antropología. Trayectorias profesionales.

An anthropologist in the museum: reflections from the experience of the professionalization of anthropology in Colombian museums

abstract The presence of anthropology in museums has a long tradition, both in the hegemonic and subaltern traditions of the discipline. Since the late twentieth century, the treatment of collections of "the others" of modernity, historically linked to the expertise of anthropologists, has been the subject of critical questioning from different places, both academic and non-academic. This text presents a personal view of these issues in order to propose a specific way of doing and thinking about anthropology in museums.

keywords Museums. Professionalization of anthropology. professional trajectories.

Um antropólogo no museu: reflexões a partir da experiência da profissionalização da antropologia nos museus colombianos

resumo A presença da antropologia nos museus tem um longo histórico, tanto na tradição hegemônica quanto na subalterna da disciplina. Desde o final do século $\mathrm{XX}$, o tratamento das coleções dos "outros" da modernidade, historicamente ligado à perícia dos 
antropólogos, tem sido sujeito a questionamentos críticos de diferentes lugares, acadêmicos e não-acadêmicos. Este texto apresenta um olhar pessoal sobre estas questões a fim de propor uma forma específica de fazer e pensar a antropologia nos museus.

palavras-chaves Museus. Profissionalização da Antropologia. Trajetórias profissionais.

\section{Preliminares ${ }^{1}$}

La antropología es una disciplina del saber indisolublemente ligada al museo, si bien una y otro tienen desarrollos diferenciados en el tiempo y en el espacio: mientras que ella aparece, con timidez, en las discusiones sobre lo que significa ser humano durante el siglo XIX, él se puede rastrear, con todas las transformaciones y matices pertinentes, desde los albores de la primera era imperial de Europa en el siglo XVI. El perfil especializado de la antropología se definió a partir del recorte que la disciplina hizo sobre cierto conjunto de objetos y materiales, ya presentes en las primeras colecciones de los gabinetes de curiosidades: aquellos que le permitirían identificar a la humanidad tanto en su unidad natural como en su diversidad histórica y cultural. Antes del gran mito del antropólogo como trabajador de campo, estuvo el gran mito del antropólogo como curador supremo. A través de los objetos, de su conservación y de su exhibición, la antropología no solo buscaba responder a una de las grandes preguntas de todos los tiempos (¿qué es, en definitiva, el ser que es el ser humano?), sino que también indagaba por sus propios contornos. Las colecciones de objetos fueron para la antropología un espejo que le devolvió una imagen coherente de sí misma, una imagen que ella aún no había articulado por completo.

La curaduría de la antropología estableció dos grandes divisiones en el inmenso universo de cosas que se pueden encontrar en el acervo de los museos: por un lado, todo lo que tenga un origen antrópico; por otro lado, todo lo que carezca de ese origen. Los dos grandes edificios de la universidad moderna fueron los que se ocuparon de esos conjuntos: las ciencias de la humanidad y las ciencias de la naturaleza. Si los primeros cien años de la disciplina fueron invertidos en levantar la gran muralla que fijara esa separación, es probable que sus próximos cien años sean testigos de su tortuoso y lento desmantelamiento. Los materiales misceláneos que componen el primer gran conjunto de cosas, el de las evidencias que cuentan el paso de la humanidad por el planeta, requirieron, a su vez, su propia gran división. La antropología se

\footnotetext{
${ }^{1}$ El texto que presento a continuación no representa la posición de la institución para la que trabajo. Sin embargo, habla de ella y desde ella. ¿Qué significa, entonces, no hablar en representación de ella? Exactamente eso: que mis palabras no las escribo porque las suscriba la institución, sino porque las suscribo yo. Es un poco extraño tener que escribir esto, pero también es un recordatorio: las personas no hablan por las instituciones y las instituciones no remplazan a las personas.
} 
apropió entonces de la gran fractura: entroncada en el nicho del salvaje, ese lugar asignado a los otros de (y por) la modernidad, definió las dos grandes colecciones de origen humano: las de nosotros (el nosotros moderno, civilizado y occidental) y las de los otros (el otros pre-moderno, salvaje, no-occidental) (Trouillot, 2011). Una fractura que alteró por completo la percepción que la disciplina tenía del mundo: sus sujetos y objetos de estudio pertenecen a un tiempo y a un espacio que no comparten con el investigador; es la negación de la coetaneidad (Fabian, 2019).

De la gran fractura se derivan luego todas las ramas del saber humanístico moderno: la historia, la sociología, las artes y la literatura, así como las especializaciones eruditas, como la filología clásica y la oriental, la etnología, el folclor o las ciencias de la religión. Todas ellas obedecen al proceso, siempre constitutivo y simultáneo, del orden moderno. No es, como usualmente se piensa, que la modernidad haya creado al salvaje para entenderse a sí misma, sino que el lugar del salvaje es el que hace posible, en primer lugar, que surja la modernidad. El espíritu moderno/colonial de la antropología no es, en consecuencia, muy reciente. Se remonta, precisamente como se remonta la historia de las colecciones y de los museos, al siglo XVI, al amanecer de la primera era imperial de Europa. La pregunta que es pertinente hoy, sin embargo, no tiene que ver con deshacer el entramado moderno/colonial del siglo XVI, sino sobre su aparición, presencia y reproducción en el siglo XXI. Al entender y asumir a la antropología como entroncada en esa historia, resulta inevitable preguntarse por el sentido de los antropólogos en el museo. Queda claro, a su vez, que la antropología es casi que inevitable en los museos: su pregunta, que es la pregunta por el sentido de la humanidad, está en la base de la configuración de las colecciones y de los museos. Si los museos y la antropología han de seguir existiendo, ¿qué significa ahora esa existencia?

\section{Un antropólogo en el museo: profesionalización de la disciplina}

La antropología cuenta con una interesante tradición académica y no académica en Colombia, donde siempre ha gozado de una significativa relevancia pública que no comparte, de la misma manera, con otras ciencias humanas en el país. Sin duda, esto tiene que ver con que la institucionalización de la antropología en Colombia estuvo vinculada al gobierno de la diferencia cultural (y, en particular, de los pueblos indígenas). La antropología comenzó su disciplinamiento institucional en Colombia en entidades de educación de carácter público, instituciones que, de alguna u otra manera, estaban articuladas al desarrollo del gobierno en Colombia. Es solo a mediados de la década de los años sesenta del siglo XX que encontramos una antropología explícitamente desvinculada del ejercicio práctico del gobierno de la diferencia cultural indígena: a su presencia y articulación en las instituciones estatales se sumará, en adelante, su institucionalización como materia de enseñanza superior en las universidades. Si bien es cierto que las tensiones que surgieron en ese nuevo contexto de institucionalización

venían desde los primeros años de la creación de las primeras instituciones de antropología en 
el país, también es cierto que solo con la incorporación del programa de antropología a las universidades estas tensiones se harán evidentes y productivas. Aunque la antropología no perderá su relevancia pública, su relación con el Estado y con el gobierno de la diferencia cultural indígena pasará por otros lugares.

En buena medida se puede argumentar que la relevancia pública de la antropología en Colombia está también estrechamente ligada a la importancia de las colecciones arqueológicas en la producción de una imagen de la nación desde finales del siglo XIX. La antropología salía a escena como la gran intérprete de esas colecciones, pues ella permitía diferenciar, con su énfasis en las clasificaciones estilísticas, una "cultura" de otra. Pero su labor no se reducía a un trabajo clasificatorio, sino que se llevaba hasta sus últimas consecuencias en su afán divulgador: alrededor de sus temas y de sus investigadores se organizaban, además de exposiciones, conferencias, programas de radio y de televisión, publicaciones para niños, juegos y un sinfín de productos. Ser antropólogo en Colombia implicaba así, desde muy temprano en la historia de la disciplina en el país, ser una autoridad en el conocimiento sobre las sociedades del pasado prehispánico y los pueblos indígenas del presente, y aventurarse a llevar sus interpretaciones por fuera de las paredes de la universidad y del museo.

Aunque en las preocupaciones de la antropología colombiana, la museología ha estado siempre presente, no es arriesgado señalar que esta última no ha encontrado un lugar específico de formación y profesionalización específico. $O$, mejor aún, que solamente es en la profesionalización donde la antropología converge en toda ley con la museología. Mucho más cerca de las preocupaciones de arquitectos, artistas e historiadores del arte, las consecuencias de la vida práctica de los museos para las colecciones arqueológicas y etnográficas (que son las que, por tradición, reclaman la presencia de antropólogos para su interpretación) no se han sentido con fuerza en la formación de la disciplina ni en sus programas de pregrado ni en sus programas de posgrado. Aun así, la antropología colombiana nunca ha dejado de estar presente en los museos y hoy, entrado el siglo XXI, parece que su presencia no va a dejar de consolidarse.

\section{Romper el consenso multicultural}

El Museo del Oro es una institución singular en el panorama de la gestión cultural en Colombia. Tiene su sede principal en Bogotá, la capital de la República, y salas de exposición permanente a su cargo en otras seis ciudades (Cartagena, Santa Marta, Armenia, Cali, Pasto y Leticia). Es un museo del Establecimiento, si bien esto requiere alguna modulación. El Museo hace parte del Banco de la República, la banca central del país, y, por lo tanto, no pertenece a las ramas ejecutivas del poder público en Colombia. La gran mayoría de instituciones públicas dedicadas a la cultura dependen de los poderes ejecutivos locales o nacionales, en cabeza del Ministerio de Cultura; en consecuencia, obedecen no solo a las lógicas electorales, sino a la voluntad política de turno. El Banco de la República tiene una autonomía especial en el 
entramado institucional que diseñó la Constitución Política de 1991, pero, de manera más significativa aún, su autonomía no es solo de derecho, sino de hecho. Pese a múltiples intentos, algunos explícitos y otros no tanto, la banca central ha mantenido su independencia y su criterio técnico. No se trata de una alabanza, sino de una constatación. El hecho de que mantenga su independencia y su criterio técnico tan solo indica que escapa a las coyunturas electorales y políticas, pero aun así es una institución que permite la reproducción del orden establecido.

La historia de la banca central del Estado, por supuesto, no puede estar por fuera del Establecimiento. El Banco de la República se crea en 1923 y su colección de orfebrería inicia en 1939. La mitología de la institución se ha encargado de divulgar la historia según la cual la colección inicia con la compra del llamado Poporo Quimbaya, un artefacto de orfebrería prehispánica identificado como perteneciente al estilo orfebre Quimbaya Temprano o Clásico que se ha definido como propio de la cuenca media del río Cauca en los primeros quinientos años de nuestra era. Un razonamiento diferente, con las mismas pruebas, es igualmente plausible: antes de la compra de ese objeto, sin duda emblemático y clave en la historia de la colección, ya se habían adquirido y conservado otras catorce piezas de orfebrería; inmediatamente después de la compra del Poporo, en 1940, se compran unos cuantos objetos más; solo en 1941, con la adquisición de varias colecciones privadas se ve expresada la racionalidad coleccionista del Banco (Uribe Villegas y García Botero, 2019). En cualquier caso, la conformación de la colección antecede por varios años a la creación de un verdadero museo, si bien en varias publicaciones se hace referencia a la colección como "museo" (García Roldán, 2020).

Desde entonces, tanto la colección de orfebrería como el Museo del Oro se convertirán en referentes centrales para la divulgación del conocimiento sobre el pasado prehispánico de Colombia. Durante más de cincuenta años, entre la apertura al público de las salas de exposición en los años cuarenta del siglo XX y la renovación del edificio y de toda la exposición permanente durante la primera década del siglo XXI, las actividades divulgativas del Museo ayudarán a consolidar dos ideas centrales para la construcción de la comunidad imaginada colombiana: el pasado autóctono de la nación antecede por varios siglos a la llegada de los europeos (es, en consecuencia, prehispánico) y (algunos de) los pueblos que habitaron el territorio de la actual Colombia dejaron evidencias (como la orfebrería) de haber sido inteligentes, cultos y civilizados. A partir de esas dos ideas se alimentaba la imagen central de la conformación cultural de la nacionalidad colombiana: su naturaleza mestiza, heredera de manera simultánea e indiferenciada de (cierta) herencia prehispánica y de (cierta) herencia post-hispánica.

En Colombia, la Constitución Política de 1991 llevó a sus límites estos argumentos. Ella no solo re-imaginó el diseño institucional del Estado colombiano, con el cual se garantizó la autonomía del Banco, sino que también propuso al multiculturalismo como una nueva forma de entender el presente y, por esa vía, el pasado del país. En el nuevo presente multicultural, el 
mestizaje era una idea problemática porque anulaba la diferencia cultural: el mestizaje significaba, también, la erradicación biológica y cultural de la diversidad étnica de la nación. Se hacía indispensable recuperar para la comunidad imaginada la diversidad que había hecho posible al mestizaje: allí donde antes se habían ejecutado políticas de exterminio y de asimilación, era necesario diseñar programas de conservación y exaltación.

Para el Museo del Oro, el régimen multicultural supuso su mayor éxito y su mayor amenaza. Éxito porque no cabe duda de que a través de él (si bien no solo por él), un público heterogéneo reconoce que en Colombia hay un pasado prehispánico que es constitutivo de la historia nacional. La colección de orfebrería, su exhibición permanente, las actividades de divulgación a su alrededor, las constantes alabanzas en el exterior, son en buena medida responsables de ese reconocimiento público. Sin embargo, con la eliminación del mestizaje de la comprensión normativa de la comunidad imaginada nacional, al Museo del Oro se le ha pedido, también, ser el museo de los otros en el multiculturalismo: el museo de los indígenas, de los afrodescendientes, de los Rom, de los raizales y, en general, de todas las diferencias étnicamente marcadas.

¿Por qué habría de ser esto una amenaza? ¿No es, precisamente, una señal indiscutible de su éxito que a través de su colección sea posible valorar públicamente la diversidad cultural de la Nación en todas sus manifestaciones, incluso más allá de las que se pueden considerar como directamente relacionadas con su colección?

Bastará recordar aquí lo que la antropología hegemónica y universitaria ha enseñado en los últimos treinta años: al sancionar política y económicamente la existencia de culturas diversas y diferentes, perfectamente delimitadas y diferenciadas, el régimen multicultural mistifica la naturaleza de las relaciones humanas. Allí donde conviven comunidades indígenas, campesinas, afrodescendientes y "occidentales" (que es preciso marcarlo entre comillas, pues es, paradójicamente, la afiliación cultural más ambigua de todas), el régimen multicultural crea emplazamientos para que cada una viva en su mundo, sin contacto, con sus tradiciones inmutables desde el principio de los tiempos. No es necesario ser antropólogo para reconocer lo inútil de esta aproximación: en cualquier lugar la gente convive y lo hace tanto en virtud como a pesar de esas adscripciones culturales.

El problema para el Museo del Oro, y por esa vía para la antropología pública y, en particular, la que tiene que hacer de la curaduría de las colecciones de los otros de la modernidad, es que ha sido encargado de asegurar el consenso multicultural (también hasta cierto punto ha asumido esta responsabilidad). Para un antropólogo en el museo el primer desafío teórico, político y práctico es desmantelar ese consenso. Allí donde se insista en la importancia de que el museo hable de los otros de la modernidad, se debe recuperar la ambición de hablar de la humanidad; allí donde se piense que la interpretación del pasado prehispánico debe contar con múltiples voces, se debe reconocer y exponer la existencia de mundos inconmensurables y no 
de realidades relativas que dependan de los puntos de vista; allí donde se invoque la leyenda rosa y la leyenda negra de la Conquista (los europeos trajeron la civilización; los europeos eran los verdaderos salvajes), se debe estudiar y poner en público la complejidad de la historia, su inagotable contingencia, su carácter impredecible .

Tras la ruptura del consenso multicultural se hará posible, y visible, el siguiente gran desafío teórico, político y práctico de un antropólogo en el museo: el de poner en escena una antropología post-humana, una antropología más allá del anthropos. No es este el lugar para hacer un ensayo bibliográfico sobre el tema; bastará con señalar que antropólogos y pensadores no-antropólogos contemporáneos vienen adelantando, en distintos lugares, una hipótesis aparentemente contraintuitiva: lo humano realmente nunca ha existido. Si romper el consenso multicultural es ir en contra de la gran fractura que la antropología impuso a las colecciones antrópicas al dividirlas en las de "nosotros" y "los otros", el siguiente paso es abordar la división entre naturaleza y cultura. No solo nunca fuimos modernos (Latour, 2007), sino que nunca fuimos humanos (Haraway, 2019; Mol, 2002). Y nunca fuimos humanos porque, entre otras cosas, antes de serlo nunca dejamos de ser seres vivos (Ingold, 2000). Y como los seres vivos existen más allá de lo que las ciencias de la naturaleza identifican como lo vivo no hay razón para conformarse con que lo humano es lo que esas mismas ciencias han definido como tal (Descola, 2012; Viveiros de Castro, 2010).

La curaduría es para el antropólogo en el museo el lugar ideal y privilegiado para explorar las consecuencias de una antropología post-humana. Si en alguna época el temor del antropólogo en el museo era caer en el fetichismo del objeto, una antropología post-humana resitúa al objeto y a la materialidad en el centro de lo que da sentido al ser humano. En pocos lugares se ha expresado esto con tanta claridad como en la paleoantropología: si se quiere exhibir la evolución de Homo sapiens no basta con exhibir la transformación de la anatomía de su género (Homo): habrá que incluir, desde su especie más antigua (Homo habilis), a sus herramientas (Arsuaga, 2019). Y si se incluyen las herramientas, ¿qué decir de los suelos transformados, los animales domesticados, las semillas dispersadas y todo el sinfín de sustancias, seres, organismos y entidades que han poblado el mundo de y con los humanos? El antropólogo en el museo debe ver esa red en los objetos que están a su cargo: la curaduría es la reparación de esa red.

Salir del régimen multicultural le permitirá al antropólogo en el museo hacer curadurías que, de nuevo, se interroguen por el sentido de lo humano. Lejos de asumir la unidad biológica de la especie como horizonte de su interrogación, lo humano aparece también como una configuración que debe ensamblarse. Las preguntas en las colecciones arqueológicas y etnográficas no son ya acerca de modos culturalmente específicos de ser humano (distantes en el tiempo y en el espacio), sino por los modos en los que ha sido posible ser humano. De esa manera, siempre es una pregunta también por el presente: de qué maneras somos humanos hoy. 


\section{Descentrar la curaduría y la antropología}

A lo largo de estas reflexiones fragmentarias, se han mantenido la antropología y la curaduría como dos personajes protagonistas. Quisiera señalar, sin embargo, que no hay nada excepcional en la antropología que la haga indispensable para los museos ni, tampoco, nada excepcional en la curaduría para pensar que es a través de ella que los museos pueden reflexionar. La defensa de la antropología como una disciplina singular contribuye a la esencialización y parcelación de los conocimientos. No solo la antropología, sino tantos y tantos nichos de la academia moderna, deben ser superados en la profesionalización, si bien en la formación universitaria es prácticamente imposible. A medida que avanzamos en las distintas jornadas y trayectorias profesionales de la antropología es posible percibir dos caminos gestados en una falsa disyuntiva: o afirmamos por completo la especificidad de la antropología y de allí sus bondades para los mundos laborales en los que participa o negamos por completo su pertinencia. La disyuntiva es falsa porque sigue situando la cuestión en el mundo de las disciplinas: un mundo parcial y parcializado, un mundo falsamente desarticulado y que exige falsamente volver a ser articulado (Wolf, 2005). Lo sabemos desde las críticas más tempranas, en el interior de la modernidad, a la especialización disciplinar. Ahora, en los albores de un nuevo mundo, que tal vez no sea sino el regreso a uno más antiguo, los llamados a la trans- y a la interdisciplinariedad son solo distracciones, planteamientos que nos exigen aferrarnos a "nuestra" disciplina para hacer valer "nuestro" conocimiento. No hay tal: ningún conocimiento le pertenece exclusivamente a alguien y, mucho menos, podemos decir que le pertenece exclusivamente a una disciplina. Los argumentos que luchan por establecer que ser antropólogo ofrece una "sensibilidad", una "manera de ver" particular, suenan hoy más impertinentes que nunca. Todo eso se puede aprender, es cierto, en una carrera de antropología, pero también (y a veces mejor) en lo que se enseña en los departamentos de filosofía, de literatura, de sociología, de historia. Pero, al final, no se trata de cambiar de carrera: se trata de aprender a dejarla atrás para volver a la pregunta que se encuentra una y otra vez en todos los lugares y en todos los tiempos: ¿qué hace posible la existencia del ser que es el ser humano?

El problema de la antropología como disciplina es que su imagen pública es mucho más coherente y nítida de lo que en realidad se deduce de sus propias conversaciones, convicciones y posturas. Allí donde muchos antropólogos están dispuestos a dudar, allí es donde se espera que aparezcan con su saber experto, sólido, indiscutible. No se me ocurre un campo más problemático para esta aparición que la participación de objetos arqueológicos y etnográficos en las exposiciones de arte. Una vez más, como ya le ocurriera en los albores del modernismo, se espera que la antropología traiga al museo y a la exposición de arte la certeza de la existencia del salvaje (Geismar, 2015). Por supuesto, el registro ha cambiado y la curaduría de arte se hace ahora preguntas que no son necesariamente idénticas a las preguntas modernas. Pero el lugar de la voz de la antropología sigue siendo convocado, y en ocasiones reivindicado, como el de la voz 
de la alteridad, el del conocimiento experto sobre la diferencia cultural. Y mientras la discusión académica, esa que se da en el seno de la disciplina institucionalizada, cuestiona ese fundamento repetidamente, el espacio público sigue demandando que se le hable de las "culturas", de "los pueblos", de "los indígenas" - pero nunca de la desigualdad, del poder o de la corrupción, temas a los que la antropología se viene dedicando desde hace varias décadas.

Si de la antropología no se puede esperar ninguna singularidad, la curaduría es solamente relevante como punto de discusión por su indiscutible visibilidad en la fabricación de los productos distintivos de los museos: las exposiciones. Pero como señalé en el acápite anterior, no es allí donde el trabajo del antropólogo -y, en realidad, el trabajo del museo- se desarrolla. La curaduría no es la última palabra y, en ese sentido, la pregunta por su autoridad es absolutamente relevante. Como ha sido señalado por una larga tradición de pensamiento hermenéutico, la autoría del conocimiento debe ser fuertemente cuestionada. El rumor de universalidad que nos llega desde distintos productos humanos y no-humanos no viene de una persona, de un autor: es el rumor del saber que se desplaza, insensible a la contingencia de las sociedades, en cada uno de los elementos que componen el mundo. Esta certeza contradice cualquier práctica profesional. Se necesita, y se exige, que se firmen los textos, que se firmen las exposiciones: es preciso apuntalar referentes, tanto para guiarnos como para rechazarlos. Pero, al hacer curaduría, cualquiera puede notar que lo que se produce está lejos de pertenecer a alguien: desde lo que se dice -los datos, la información, si se quiere- hasta cómo se dice -los objetos seleccionados, su exhibición, las luces de la sala- no hay una autoría identificable en una persona singular. El curador entra a participar en una red en la que se distribuye la agencia de su propio conocimiento y de la producción de la exposición. La renuncia a la autoría es también la renuncia al protagonismo. ¿Quiere decir ello una renuncia a la curaduría? Creo que no. Creo que el que ocupe el papel del curador debe saber que es una pieza más en un ensamblaje que, él mismo, debe ser objeto de su cuidado y de su atención, tanto o más que los objetos. Porque la renuncia a la autoría no puede ser una renuncia a la responsabilidad de lo que se dice y de lo que se quiere hacer decir: pero esa responsabilidad no se fija en un cargo, sino en una labor.

\section{Hacia una política de los pequeños gestos}

La curaduría, como dice Michel Foucault de la genealogía, es gris, meticulosa. Las exposiciones son solo un momento minúsculo de la cotidianidad del trabajo en el museo. Es un momento fastuoso, naturalmente. En cada inauguración el museo tiene la oportunidad de mostrarse como producto, más que como proceso. En sus entrañas, todos quienes lo habitan y lo conforman saben que la inauguración de la exposición no quiere decir que la exposición se haya terminado, sino que se abrió al público. La exposición, ella misma, es un proceso, un organismo que también llegará a su final. 
Como eventos fastuosos, las exposiciones y sus inauguraciones son altamente efectistas. El antropólogo en el museo debe huir de ellas y concentrarse en lo que la etnografía le ha enseñado a concentrarse: el devenir cotidiano del mundo, que es, en realidad, en donde el mundo se hace mundo. Los museos necesitan urgentemente una política de los pequeños gestos: menos palabras grandilocuentes (descolonizar, democratizar, reimaginar, reinventar) y más acciones humildes. Si el antropólogo está en el museo debe empezar por reparar las fracturas sobre las cuales se ha fundado su disciplina. Para eso debe, en primer lugar, reconocerlas. En los museos puede ser difícil y puede requerir años de trabajo (y ese trabajo es, en sí mismo, uno de esos pequeños gestos). Pero hay situaciones que no pueden ser toleradas más y en las cuales el antropólogo debe actuar, si bien no vengan con sus acciones grandes celebraciones. Actuar contra ellas es una acumulación de pequeños gestos para reescribir la muerte que vive en los museos (Hicks, 2021).

En primer lugar, reescribir la muerte de los seres humanos. La exhibición de restos óseos es altamente problemática. Mi propia posición, sin embargo, es simple, con lo que no pretendo zanjar ni la discusión ni sus consecuencias: estos deben ser retirados de manera permanente de la exhibición, incluso allí (y tal vez sobre todo allí) donde su exhibición sea un "gancho" para atraer a los públicos o donde no haya reclamaciones formales por un grupo específico de personas. Al ser retirados de la exhibición, el museo debe velar por otorgarles un destino final a esos restos, ya sea en el propio museo o por fuera de él. Aunque los restos óseos descansen para siempre en el museo, deben ser excluidos de las colecciones: no se les puede seguir inventariando, midiendo e interviniendo como si se tratara de cualquier otra cosa. Si bien es cierto que en diferentes regímenes ontológicos las cosas pueden estar tan vivas como los humanos, también es cierto que no están vivas de la misma manera que los humanos y, en consecuencia, el trato que se le da a un artefacto no es idéntico al que se les da a los restos mortales de una persona.

En segundo lugar, también se debe reescribir la muerte de los objetos. Según la información transmitida por los arqueólogos o por las personas que servían de intermediarios entre los que encontraban los objetos y el Museo del Oro, un elevado porcentaje de objetos viene de contextos de enterramiento, es decir, de contextos que fueron creados por personas para el cuidado de los restos mortales de otras personas. Lo que luego se traduce en las clasificaciones y tipologías técnicas de la arqueología y de la exhibición oculta el hecho de que se trata de objetos de muertos, de objetos para los muertos. Allí donde no existen reclamaciones para la restitución de esos objetos, el antropólogo en el museo debe pensar qué significa su exhibición y si el sentido de su exhibición puede ser justificable. La exhibición no es independiente de la adquisición de los objetos: la exhibición es una repetición y una continuación en el presente del acto mismo de la extracción (Hicks, 2021). Es probable que las acciones de estas reescrituras sean imperceptibles: que no haya grandes exposiciones ni grandes discursos ni celebraciones; a lo 
mejor tan solo se trata de ajustar una vitrina, de diseñar un espacio para los muertos y sus objetos en un lugar inaccesible para el público, de inventar nuevos dispositivos de comunicación y de indagación. Pero lo más probable es que se trate de acciones complejas que requieren no solo voluntad, sino largos procesos de conversación, consulta, prueba y error.

En tercer lugar, la reescritura de la muerte debe codificarse en la base de datos del museo. El acervo de información que constituye la colección es un elemento crucial para la reescritura de la muerte. La idea de una base de datos tipológica (¿qué son estos objetos?) es a todas luces insuficiente por reduccionista. Cualquier antropólogo en el museo sabe que un objeto no es aquello que es la función del objeto. Por el contrario, el objeto es su propia vida y muerte, tanto por fuera como en el museo. El registro de las colecciones es, entonces, la consolidación de los materiales que hacen posible reescribir la muerte que vive en el museo: voces, manuscritos, exámenes, confesiones, textos científicos, reclamaciones legales... La colección no es el objeto: es la red de sentido que hace que exista aquí y ahora. Siempre considerada como un ámbito eminentemente técnico, el registro de la colección pasa ahora a ser un ámbito de intervención ontológica (¿qué ser es este objeto?).

\section{Cierre}

No me gusta mucho la imagen del revolucionario que dice que lucha por cambiar el sistema desde adentro: por una parte, porque trivializa la revolución, que es una transformación del orden establecido y, por otra parte, porque adormece la conciencia crítica del individuo, que encuentra en esa expresión un triste, pero reconfortante consuelo para llevar a cabo su labor como miembro de una institución. El trabajo del antropólogo en el museo enfrenta desafíos de diferente escala, magnitud y alcance, y en todas ellas debe enfrentarse a la innegable realidad de trabajar para un tipo de institución cuya vocación es reificar el orden establecido (y hablo aquí no solo de los llamados museos hegemónicos, sino de cualquier forma de musealización). Pero justamente por eso, la actividad profesional de la disciplina en el museo se parece más a una maratón -con vallas, si tal cosa existiese- que a una carrera de cien metros planos. La constancia en el ritmo solo se logra recorriendo varias veces el camino de la competencia. De esa manera se aprenden las formas y los tiempos del ataque y de la defensa, y también se identifica que la paciencia puede ser una mejor compañía que la fuerza.

El antropólogo en el museo no puede renunciar a hacer una cartografía de los malestares, pero tampoco puede pretender que toda su labor se reduzca a hacer esa cartografía. De manera simultánea, debe también participar en la vida cotidiana de la institución, aprender de ella, reconocer sus límites y sus contradicciones. Eso implica no solo ejecutar una práctica teóricamente informada, sino llevar una teoría prácticamente inspirada. Hay que saber participar de la burocracia, pero también hay que saber imaginar un mundo sin ella; hay que involucrarse en la gestión, pero también saber que las relaciones sociales no se agotan en ella. 
En más de una ocasión, el antropólogo en el museo encontrará con más claridad los obstáculos que las oportunidades, que se le podrán aparecer en distintas formas - desde la inercia institucional hasta las expectativas públicas acerca de su labor. Y allí, en esa encrucijada, lo único de lo que podrá valerse, al igual que el maratonista, es de su experiencia: de haber recorrido, una y otra vez, el mismo camino, sin por ello estar obligado a recorrerlo siempre de la misma manera.

\section{Referencias}

ARSUAGA, Juan Luis. 2019. Vida, la gran historia. Un viaje por el laberinto de la evolución. Madrid: Crítica.

DESCOLA, Philippe. 2012 [2005]. Más allá de naturaleza y cultura. Traducción de Horacio Pons. Buenos Aires: Amorrortu.

FABIAN, Johannes. 2019 [1983]. El tiempo y el Otro. Cómo construye su objeto la antropología. Traducción de Cristóbal Gnecco. Bogotá: Universidad de los Andes; Universidad del Cauca. GARCÍA ROLDÁN, Daniel. 2020. El museo vivo y el museo imaginario. Reproductibilidad y medios de comunicación de masas en los museos arqueológicos colombianos: prácticas globales y apropiaciones locales (1938-1968). In: Nuevo Mundo, Mundos Nuevos [En línea], Extracto de tesis, puesto en línea el 8 de octubre de 2020, consultado el 13 de junio de 2021. DOI: https://doi.org/10.4000/nuevomundo.81937

GEISMAR, Haidy. 2015. "The Art of Anthropology: Questioning Contemporary Art in Ethnographic Display”. In: WITCOMB, Andrea; MESSAGE, Kylie; (Eds). The International Handbooks of Museum Studies. Volume 1: Museum Theory. Wiley-Blackwell. DOI: https://doi.org/10.1002/9781118829059.wbihms110

GNECCO, Cristóbal. 2017. Antidecálogo. Diez ensayos (casi) arqueológicos. Popayán: Universidad del Cauca.

HARAWAY, Donna. 2019 [2016]. Seguir con el problema: Generar parentesco en el Chthuluceno. Traducción de Helen Torres. Bilbao: Consonni.

HICKS, Dan. 2021. Necrography: Death-Writing in the Colonial Museum. In: British Art Studies, n.19. DOI https://doi.org/10.17658/issn.2058-5462/issue-19/conversation

INGOLD, Tim. 2000. The perception of the environment. Essays on livelihood, dwelling and skill. London: Routledge.

LATOUR, Bruno. 2007 [1991]. Nunca fuimos modernos. Ensayo de antropología simétrica. Traducción de Víctor Goldstein. Buenos Aires: Siglo XXI.

MOL, Annemarie. 2002. The Body Multiple: ontology in medical practice. Londres: Duke University Press.

TROUILLOT, Michel-Rolph. 2011 [2003]. Transformaciones globales. La antropología y el mundo moderno. Traducción de Cristóbal Gnecco. Bogotá: Universidad de los Andes; Universidad del Cauca. 
URIBE VILLEGAS, María Alicia; GARCÍA BOTERO, Héctor. 2019. Conformación de la colección prehispánica del Museo del Oro del Banco de la República (Bogotá): Contextos, tendencias y aperturas, 1936-2019. In: Revista Goya, n. 367: p. 124-139.

VIVEIROS DE CASTRO, Eduardo. 2010 [2009]. Metafisicas caníbales. Líneas de antropología postestructural. Traducción de Stella Mastrangelo. Buenos Aires: Katz.

WOLF, Eric. 2005 [1982]. Europa y la gente sin historia. Traducción de Horacio Pons. México DF: Fondo de Cultura Económica.

sobre el autor

\section{Héctor García Botero}

Curador de Arqueología y etnografía del Museo del Oro del Banco de la República (Bogotá, Colombia). Es licenciado en Antropología y maestro en Antropología Social por la Universidad de los Andes.

Autoría: No aplicable

Financiación: La producción de este texto no contó con financiación

Fecha de recibimiento 12/04/2021

Fecha de aprobación em 22/06/2021 